\title{
Aprendizaje de los fundamentos de la probabilidad apoyado en las TICs
}

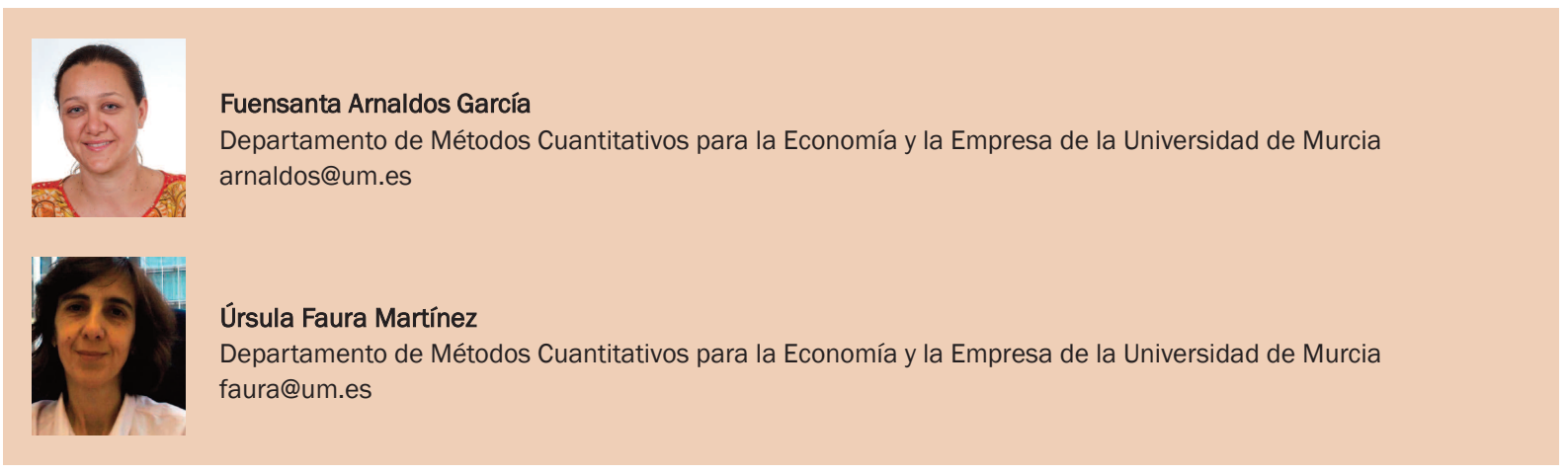

|Fecha presentación: 28/09/2011 | Aceptación: 30/05/2012 | Publicación: 20/12/2012

\section{Introducción}

Tradicionalmente la enseñanza de la Estadística en las universidades se ha realizado con escaso apoyo de las nuevas tecnologías de la información y las comunicaciones, TICs, ya que ha estado basada fundamentalmente en una transmisión de conocimientos por parte del docente. Pero, ante el reto de adaptación al nuevo Espacio Europeo de Educación Superior (EEES), la nueva configuración de los planes de estudios ha propiciado un proceso de innovación en las estrategias formativas. Ahora, además de transmitir conocimientos, el profesor debe actuar como motivador y guía, para que el alumno descubra nuevas formas de aprendizaje. Por su parte el alumno debe gestionar su proceso de aprendizaje y adquirir la capacidad de comprender y analizar los conceptos dados. En este contexto, supone un gran avance apoyarse en las nuevas tecnologías.

El uso de la tecnología como una herramienta más dentro de la clase permite desarrollar nuevas experiencias formativas y es una motivación extra para el alumno que afronta la asignatura con otra perspectiva, mucho más atractiva para él. En este sentido, dado el elevado número de recursos a los que tenemos acceso a través de la red, ésta puede considerarse una herramienta docente más ya que facilita la integración de las TICs en la docencia en asignaturas de Estadística de múltiples formas.

Los libros online o las revistas de docencia en Estadística permiten que solicitemos a los alumnos completar cualquiera de los contenidos de las asignaturas. La disponibilidad de datos, tanto en fuentes oficiales, como en páginas que los agrupan por temas o métodos de análisis, facilitan el que les pidamos realizar análisis de los mismos. Estos análisis pueden llevarlos a cabo no sólo utilizando software comercial, sino empleando cualquiera de los muchos programas de uso libre a los que podemos acceder y apoyándose también en las denominadas calculadoras estadísticas, cuyo acceso es también libre. Además, el uso de las herramientas de la Web 2.o (Blogs, Wikies, videoconferencias, redes so- ciales,...) posibilitan intercambios con el estudiante y entre estudiantes que proporcionan nuevas experiencias a incorporar al proceso de enseñanza-aprendizaje.

Entre todos estos recursos, nos gustaría destacar la presencia en la red de las denominadas simulaciones interactivas. Son representaciones gráficas e interactivas de un fenómeno, que permiten al estudiante realizar cambios y observar su efecto en el fenómeno que se representa. Tienen dos características que las hacen interesantes desde un punto de vista didáctico: la visualización de los conceptos estadísticos tradicionalmente difíciles de comprender, y las posibilidades de interacción, que permiten al estudiante experimentar con el fenómeno y aprender de la propia experiencia.

La proliferación de este tipo de recursos en la red facilita su incorporación a la docencia. Existen numerosas simulaciones disponibles para su uso, en la mayoría de los casos gratuitas y bajo licencias de uso Creative Commons. En su mayor parte se encuentran disponibles en forma de applets, bien en páginas web que pueden ser referenciadas para su uso, bien en código abierto que puede ser incorporado a una página web diferente. También es de destacar la existencia de proyectos de potenciación de la visualización desde grandes empresas como el caso de Wolfram Research o desde instituciones sin ánimo de lucro como las responsables del desarrollo de Geogebra.

Son muchos los ámbitos de la docencia en estadística en los que estas simulaciones interactivas se pueden aplicar:

- Estadística descriptiva: análisis univariante de datos, interpretación de medidas descriptivas, representación gráfica de datos, regresión lineal, etc.

- Probabilidad: visualización de sucesos y operaciones de sucesos, concepto de sucesos incompatibles, independientes, probabilidad condicionada, etc.

- Variables aleatorias: comprensión de los tipos de variables aleatorias, de la interpretación de sus principales características, de las funciones que describen su comportamiento aleatorio, etc. 
- Modelos de variables aleatorias: comprensión de la naturaleza y efecto de las variaciones paramétricas, mejorar la capacidad de distinción entre los tipos de modelos, etc. - Inferencia paramétrica y no paramétrica: interpretación de las propiedades de los estimadores, naturaleza de los tipos de estimación, estudio de conceptos como el nivel de confianza, el p-valor, potencia de un test, etc.

El objetivo principal de este trabajo es ilustrar el uso de estas simulaciones interactivas como herramientas que facilitan la asimilación y comprensión de los diferentes conceptos desarrollados en el estudio de la Estadística. Para ello, y dado el elevado número de recursos disponibles, seleccionamos una de las posibles aplicaciones: la probabilidad. Mostramos que existen numerosas simulaciones disponibles para su uso, que pueden ser referenciadas como cualquier otro recurso bibliográfico, de forma que, verificado que cumplen los objetivos planificados en el desarrollo teórico del tema, pueden integrarse en las actividades diseñadas por los docentes tanto para su uso en el aula como fuera de ella. Finalmente, ofrecemos una propuesta de integración de las simulaciones en una actividad práctica.

\section{Simulaciones en Estadística}

En la Tabla 1 mostramos algunos proyectos desarrollados que incorporan la visualización y que pueden servir de fuente de información y punto de partida si se desea incluir este tipo de recursos en la docencia. Son también destacables por su utilidad y calidad páginas personales de autores

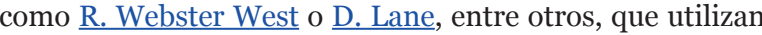
estos recursos desde hace muchos años y han participado en la difusión de los mismos.

Una revisión de los enlaces citados puede dar una idea de los recursos disponibles y su uso potencial. Adicionalmente, y para un tema concreto, puede completarse la revisión con la realización de búsquedas sistemáticas en revistas de docencia en estadística, como Journal of Statistics Edu-

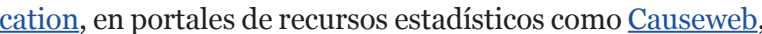
o en buscadores de uso general como Google.

\section{Simulaciones para Probabilidad}

En el estudio de la probabilidad nos planteamos distintos objetivos, que perseguimos a través del uso de diferentes estrategias docentes:

A. Comprensión de los sucesos, las operaciones con los mismos y las consecuencias de los axiomas de Kolmogorov.

B. Cálculo de probabilidades de sucesos individuales o de operaciones de sucesos mediante la interpretación clásica de la probabilidad.

C. Comprensión del significado de la interpretación frecuentista de la probabilidad.

D. Interpretación del concepto de probabilidad condicionada y su relación con la probabilidad de la intersección de sucesos.

E. Planteamiento de los experimentos en etapas y el uso de los teoremas de la Probabilidad Total y Bayes para su resolución.

F. Conexión entre el tema de probabilidad en el que trabajan con sucesos y el concepto de variable aleatoria.

En la consecución de estos objetivos somos conscientes de las dificultades que tienen habitualmente nuestros alumnos y, con el propósito de facilitar la comprensión de los conceptos planteados y permitirles aprender de su propia experiencia, exponemos algunas simulaciones que pueden ayudar a resolver esas dudas y complementar el desarrollo tradicional de las clases.

A. Comprensión de los sucesos y las operaciones con los mismos

Tradicionalmente la representación gráfica a través de diagramas de Venn ha resultado muy útil para entender mejor cualquier concepto relacionado con sucesos. El problema del que adolece la presentación tradicional es que los alumnos no pueden experimentar por su cuenta con unos gráficos ya hechos. Hay nociones como la incompatibilidad, las leyes de Morgan o el trabajar con más de dos sucesos, que les pueden quedar mucho más claros mediante la experimentación. En el applet de Shepperd (Sim1) se muestran gráficamente 16 posibles operaciones entre dos sucesos que se eligen en un menú. La operación contraria, esto es, seleccionar los sucesos y observar su notación puede realizarse en el applet de Brodie (Sim2) del proyecto de Demostración Wolfram tanto para dos, como para tres sucesos. En el mismo proyecto, Beck y Kent (Sim3), ofrecen otro applet en el que se representan gráficamente y a partir de la notación habitual 127 operaciones con tres sucesos (ver Tabla 2).

Las simulaciones 4 a 6 implican los mismos conceptos pero incorporan también la probabilidad del suceso interpretándose la misma como la proporción que sobre el espacio muestral (caja completa) supone el espacio que ocupa el suceso. Estas tres simulaciones permiten trabajar conceptos como la unión de sucesos disjuntos, la inclusión de sucesos y propiedades como la distributiva o las leyes de Morgan, así como experimentar con las consecuencias de los axiomas de Kolmogorov. Cada uno de estos applets presenta peculiaridades. El applet de West (Sim4) no permite alterar las probabilidades de los sucesos A y B y se centra en estudiar los efectos de los cambios en la relación entre los sucesos. El applet de Stark (Sim5) trabaja con tres sucesos y permite modificar tanto las probabilidades como la relación entre ellos. El mismo autor presenta otro applet Venn diagrams (2 subsets) similar al de West que sí permite modificar probabilidades. Por su parte, el applet de Borrego (Sim6), del aula virtual de Estadística, ofrece la posibilidad de trabajar con uno, dos o tres sucesos, así como modificar tanto las probabilidades de los sucesos como la relación entre ellos.

B. Cálculo de probabilidades de sucesos individuales o de operaciones de sucesos mediante la interpretación clásica de la probabilidad.

En los applets de las simulaciones 4 a 6 la probabilidad de los sucesos se mide en función de la superficie que éstos ocupan. Introducida esta idea, el trabajar con experimentos sencillos permite utilizar la regla de Laplace para la obtención de la probabilidad de sucesos. En este sentido, los experimentos aleatorios de lanzamiento de dados o de selección de bolas en urnas, son sencillos y fáciles de entender por los alumnos. El applet del proyecto Statmedia (Sim7) calcula la probabilidad de cualquier suceso que podamos plantear en el lanzamiento de un dado. Puede complementarse con el applet de Barrow (Sim8) que considera el experimento de lanzamiento de dos dados. Respecto a los experimentos de urnas, el proyecto Statmedia incorpora un applet (Sim9) que trabaja con la extracción sin reemplazamiento de una a tres bolas de una urna de cinco bolas de dos colores (ver Tabla 3).

C. Comprensión del significado de la interpretación frecuentista de la probabilidad.

Las simulaciones interactivas proporcionan una forma de introducir a los alumnos la interpretación frecuentista de la 


\begin{tabular}{|c|c|}
\hline PROYECTO & CONTENIDO $^{1}$ \\
\hline DEMOSTRACIÓN WOLFRAM & $\begin{array}{l}\text { Recurso de código abierto con miles de visualizaciones gratuitas tanto científi- } \\
\text { cas como educativas. Agrupadas por temas, cada visualización es accesible } \\
\text { desde el navegador en modo demostración, o de manera interactiva si se dis- } \\
\text { pone del software Mathematica o de la versión gratuita Wolfram CDF Player. } \\
\text { El código puede variarse y ofrecerse a través del proyecto. } \\
\text { Software: Wolfram CDF Player (gratuito) }\end{array}$ \\
\hline$\underline{\text { GEOGEBRA }}$ & $\begin{array}{l}\text { Recurso de código abierto con miles de visualizaciones gratuitas. Inicialmente } \\
\text { para matemáticas de Enseñanza Secundaria, en la actualidad se ofrecen simu- } \\
\text { laciones interactivas en numerosos temas. El código puede variarse y ofrecerse } \\
\text { a través del proyecto. } \\
\text { Software: Geogebra (gratuito) } \\
\text { El software es utilizado en otros proyectos de visualización en distintos niveles } \\
\text { de educación, como el proyecto Gauss del Ministerio de Educación. }\end{array}$ \\
\hline$\frac{\text { DESCARTES }}{\text { Ministerio de Educación }}$ & $\begin{array}{l}\text { Colección de materiales y unidades didácticas interactivas que cubren contenidos } \\
\text { de las matemáticas de E.S.O. y Bachillerato. Dispone de un curso destinado a pro- } \\
\text { fesores que tengan alguna experiencia con el uso del ordenador y quieran aprender } \\
\text { a configurar las escenas interactivas e insertarlas en páginas web. } \\
\text { Software: Descartes web 2.o (gratuito). Máquina virtual de Java (gratuita). }\end{array}$ \\
\hline$\frac{\text { STATMEDIA }}{\text { Universidad de Barcelona }}$ & $\begin{array}{l}\text { Materiales del grupo de innovación Statmedia del Departamento de Estadística } \\
\text { de la Universidad de Barcelona. Este grupo tiene como objetivo la mejora y la } \\
\text { innovación docente en el ámbito de la Estadística aplicada y lleva desarrollando } \\
\text { entornos multimedia basados en Java desde 1999. Los applets diseñados están } \\
\text { disponibles tanto integrados en la versión demostración online del curso Stat- } \\
\text { media I, como de manera separada. Los que no están disponibles online se en- } \\
\text { cuentran en la publicación en CD del curso. Cubren probabilidad e inferencia. } \\
\text { Software: Máquina virtual de Java (gratuita). }\end{array}$ \\
\hline $\begin{array}{l}\text { AULA VIRTUAL DE ESTADÍSTICA } \\
\text { Universidad de Córdoba }\end{array}$ & $\begin{array}{l}\text { Materiales de acceso libre disponibles en la plataforma educativa WebGeC del } \\
\text { Departamento de Estadística de la UCO. Conjunto de applets realizados en Java } \\
\text { divididos en grupos según los temas didácticos a tratar, que pertenecen a un } \\
\text { proyecto más amplio de integración de TICs en la enseñanza de la Estadística. } \\
\text { Cubren descriptiva, probabilidad e inferencia. } \\
\text { Software: Máquina virtual de Java (gratuita). }\end{array}$ \\
\hline$\underline{\text { SOCR }}$ UCLA University & $\begin{array}{l}\text { Proyecto colaborativo de recopilación de materiales de Estadística online en el que } \\
\text { participan miembros de distintos centros y Departamentos de UCLA. Repositorio } \\
\text { de applets interactivos cuyo acceso se realiza a partir de las categorías establecidas } \\
\text { en el proyecto que abarca análisis de datos, modelización y experimentación y pro- } \\
\text { porciona todo tipo de recursos educativos. } \\
\text { Software: Máquina virtual de Java (gratuita). }\end{array}$ \\
\hline$\frac{\text { WISE }}{\text { Claremont Graduate University }}$ & $\begin{array}{l}\text { Proyecto de uso de recursos online de Estadística. Applets relativos a inferencia } \\
\text { y al Teorema Central del Límite, disponibles de manera separada o insertados } \\
\text { en tutoriales dedicados al estudio de un tema apoyado en los applets y todo tipo } \\
\text { de material adicional. } \\
\text { Software: Máquina virtual de Java (gratuita). }\end{array}$ \\
\hline
\end{tabular}

Tabla 1: Proyectos destacados de visualización y experimentación con conceptos estadísticos

${ }^{1}$ Para la ejecución de los applets es necesario que esté instalada la máquina virtual Java (complemento de los navegadores que permite ejecutar programas redactados en lenguaje Java). En http://www.java.com/es/download/index.jsp se puede comprobar si está instalada y, en caso contrario, descargar una copia gratuita. Para trabajar con los ficheros del proyecto de demostración Wolfram es necesario tener instalado el Wolfram CDF Player. En http://demostrations.wolfram.com/download-cdf-player.html se puede descargar una copia gratuita. 


\begin{tabular}{|c|c|c|}
\hline \multicolumn{2}{|c|}{ SIMULACIÓN INTERACTIVA } & \multirow{2}{*}{$\begin{array}{l}\text { DESCRIPCIÓN } \\
\text { Para dos sucesos. Permite escoger entre } 16 \text { posibles operaciones utilizando } \\
\text { la notación para seleccionar. Como consecuencia de la selección se representa } \\
\text { a través de un diagrama de Venn, coloreado en rojo, el suceso elegido. }\end{array}$} \\
\hline Sim 1 & $\frac{\text { Venn Diagram Applet }}{\text { M. Shepperd }}$ & \\
\hline Sim2 & $\begin{array}{c}\text { Interactive Venn Diagrams } \\
\text { Proyecto Demostración Wolfram } \\
\text { M. Brodie }\end{array}$ & $\begin{array}{l}\text { Para dos y tres sucesos. Sobre un diagrama de Venn permite seleccionar pin- } \\
\text { chando el suceso que se desee. Como consecuencia lo sombrea en el diagrama y, } \\
\text { opcionalmente, muestra distintas notaciones equivalentes para el suceso elegido. }\end{array}$ \\
\hline Sim3 & $\begin{array}{l}\text { Venn Diagrams } \\
\text { Proyecto Demostración Wolfram } \\
\text { G. Beck y L. Kent }\end{array}$ & $\begin{array}{l}\text { Para dos sucesos. Sobre un diagrama de Venn permite seleccionar pinchando } \\
\text { hasta } 127 \text { sucesos diferentes. Como consecuencia lo sombrea en partes en el } \\
\text { diagrama y, opcionalmente, muestra la notación del suceso elegido, como } \\
\text { unión de las partes sombreadas en distintos colores. }\end{array}$ \\
\hline Sim 4 & $\frac{\text { Conditional Probability Applet }}{\text { W. West }}$ & $\begin{array}{l}\text { Para dos sucesos. Trabaja con probabilidades. Incluye el cálculo de dos pro- } \\
\text { babilidades condicionadas. Permite escoger entre } 8 \text { posibles operaciones uti- } \\
\text { lizando la notación para seleccionar. Como consecuencia de la selección se } \\
\text { representa a través de un diagrama de Venn, coloreado en verde el suceso } \\
\text { elegido. El tamaño del suceso representa su probabilidad. La probabilidad } \\
\text { de los sucesos A y B no se puede alterar, pero sí la relación entre ellos. }\end{array}$ \\
\hline Sim5 & $\begin{array}{l}\text { Venn Diagrams }(3 \text { subsets }) \\
\text { SticiGui } \\
\text { P.B. Stark }\end{array}$ & $\begin{array}{l}\text { Para tres sucesos. Trabaja con probabilidades. Incluye el cálculo de seis pro- } \\
\text { babilidades condicionadas diferentes. Permite escoger entre } 20 \text { posibles ope- } \\
\text { raciones utilizando la notación para seleccionar. Como consecuencia de la } \\
\text { selección se representa a través de un diagrama de Venn, coloreado en azul } \\
\text { el suceso elegido. El tamaño del suceso representa su probabilidad. Se puede } \\
\text { alterar tanto la probabilidad de los sucesos como la relación entre ellos. }\end{array}$ \\
\hline Sim6 & $\begin{array}{l}\text { Diagrama de Venn } \\
\text { Aula Virtual Estadística } \\
\text { J. Borrego }\end{array}$ & $\begin{array}{l}\text { Para uno, dos y tres sucesos. Trabaja con probabilidades. Incluye probabilidad } \\
\text { condicionada (distintas opciones según el número inicial de sucesos: } 2 \text { y 3). Per- } \\
\text { mite escoger } 2,12 \text { y } 18 \text { posibles operaciones utilizando la notación para seleccio- } \\
\text { nar (distintas opciones según el número inicial de sucesos). Como consecuencia } \\
\text { de la selección se representa a través de un diagrama de Venn, coloreado en gris } \\
\text { el suceso elegido. El tamaño del suceso representa su probabilidad. Se puede al- } \\
\text { terar tanto la probabilidad de los sucesos como la relación entre ellos. }\end{array}$ \\
\hline
\end{tabular}

Tabla 2: Simulaciones interactivas seleccionadas por su utilidad para la consecución del objetivo A

probabilidad que les ayuda a comprender sus implicaciones. Sustituyen a la realización de un experimento para el que no dispondríamos de tiempo, ya que con las simulaciones existentes el alumno puede experimentar y observar gráficamente los resultados del experimento.

En el applet de Lane (Sim10) se simulan tiradas sucesivas de una moneda perfecta. En él se representa gráficamente la frecuencia relativa del suceso cara de manera dinámica, conforme se va produciendo el experimento, y se encuentra ubicado en un tutorial en el que se hace énfasis en mostrar que la convergencia de la frecuencia relativa no implica igualdad en los resultados en términos de número de caras y cruces. El mismo experimento se recoge en los applets de Aguilera, en el aula virtual de Estadística (Sim11), y del proyecto Statmedia (Sim12). En el applet del aula virtual de Estadística se puede trabajar con monedas no perfectas, aunque presenta una limitación en el número de tiradas que no tiene el applet de Lane. Por su parte, el applet del pro-

\begin{tabular}{|l|c|l|}
\hline \multicolumn{2}{|c|}{ SIMULACIÓN INTERACTIVA } & DESCRIPCIÓN \\
\hline Sim7 & $\begin{array}{c}\text { C1BasicDie } \\
\text { Proyecto Statmedia }\end{array}$ & $\begin{array}{l}\text { Experimento aleatorio de lanzamiento de un dado perfecto. Permite el cálculo de la pro- } \\
\text { babilidad de todos los sucesos que se pueden plantear en el experimento a partir de la } \\
\text { visualización de los resultados favorables y de los resultados posibles. Trabaja también } \\
\text { las probabilidades condicionadas a partir de la reducción del espacio muestral. }\end{array}$ \\
\hline Sim8 & $\begin{array}{l}\text { Conditional Probabilities } \\
\text { R.F. Barrow }\end{array}$ & $\begin{array}{l}\text { Experimento aleatorio de lanzamiento de dos dados perfectos. Posibilita el cálculo de } \\
\text { la probabilidad de algunos sucesos predefinidos a partir de la visualización de los casos } \\
\text { favorables y posibles en una tabla de doble entrada y la regla de Laplace. Trabaja las } \\
\text { probabilidades condicionadas de dos formas, a través de su definición, y considerando } \\
\text { la reducción del espacio muestral. }\end{array}$ \\
\hline Sim9 & $\begin{array}{l}\text { Experimento aleatorio de extracción sin reemplazamiento en una urna. Se pueden ex- } \\
\text { traer hasta 3 bolas de un conjunto de } 5 \text { con dos posibles colores, siendo modificable } \\
\text { la composición de la urna. A partir de la visualización de los casos favorables y posibles } \\
\text { permite el cálculo de probabilidades de todos los sucesos que se pueden definir sobre } \\
\text { el número de bolas extraídas de un color (blanco). }\end{array}$ \\
\hline
\end{tabular}

Tabla 3: Simulaciones interactivas seleccionadas por su utilidad para la consecución del objetivo B 
yecto Statmedia destaca por la rapidez de uso, aunque también tiene una limitación en el número de tiradas

El applet de Savory, en el proyecto de Demostración Wolfram (Sim13) se puede observar el principio de regularidad estadística a partir del lanzamiento de un dado perfecto, mostrando los resultados de las tiradas en un diagrama de sectores. El applet de Aguilera, del aula virtual de Estadística (Sim11), permite también trabajar el experimento de lanzamiento de un dado en el que se pueden alterar las probabilidades de obtención de cada una de sus caras, siendo la representación de los resultados similar a la utilizada en el lanzamiento de una moneda. Por su parte, en el applet de Sada Allo (Sim14), los resultados de las tiradas del dado, se representan con un diagrama de barras. Por último el applet de Brown, también en el proyecto de Demostración Wolfram (Sim15), trabaja el experimento de lanzamiento de dos dados, visualizando el histograma de los resultados en término de la suma de sus puntuaciones (ver Tabla 4). D. Interpretación del concepto de probabilidad condicionada y su relación con la probabilidad de la intersección de sucesos. Cuando estudiamos la probabilidad condicionada nos encontramos con que los alumnos tienen muchas dificultades para poder sacar el máximo provecho de este concepto. Así, confunden independencia con incompatibilidad, probabilidad condicionada con probabilidad conjunta o no diferencian entre $\mathrm{P}(\mathrm{A} / \mathrm{B})$ y $\mathrm{P}(\mathrm{B} / \mathrm{A})$.

El concepto de probabilidad condicionada puede trabajarse de distintas maneras con las simulaciones interactivas.
Se puede estudiar gráficamente, y en ese sentido, los applets de West (Sim4), de Stark (Sim5), y de Borrego (Sim6), además de utilizarse para obtener probabilidades de operaciones con dos o tres sucesos apoyándose en los diagramas de Venn, están preparados para calcular probabilidades condicionadas, e interpretarlas como la proporción que ocupa el suceso intersección sobre el suceso condición y no sobre el espacio muestral. Dado que todos ellos permiten alterar la relación entre sucesos, posibilitan el trabajar con sucesos incluidos y con sucesos disjuntos, profundizando en las consecuencias sobre la probabilidad condicionada. Este enfoque gráfico puede observarse de una manera sencilla también en el applet de Boucher (Sim16), del proyecto Demostración Wolfram.

El enfoque del applet de Lane (Sim17) se centra en la reducción del espacio muestral y en el cálculo de la probabilidad condicionada utilizando la regla de Laplace bajo distintos ejemplos basados en colores y figuras diferentes. Utilizando el mismo enfoque, pero con el experimento del lanzamiento de dos dados, Richman et al (Sim18) presentan de manera gráfica los sucesos intersección y condición, lo que posibilita el cálculo y comparación de las probabilidades conjuntas y condicionadas. El applet de Barrow citado con anterioridad (Sim8) trabaja este mismo experimento de una manera similar, aunque la visualización de los resultados es diferente y los sucesos que se pueden seleccionar también. En este caso las probabilidades condicionadas se calculan de manera explícita, bien considerando la reducción del espacio muestral que se produce, bien utilizando la definición de probabilidad condicionada y calcu-

\begin{tabular}{|c|c|c|}
\hline \multicolumn{2}{|c|}{ SIMULACIÓN INTERACTIVA } & DESCRIPCIÓN \\
\hline Sim10 & $\begin{array}{l}\text { Gambler's Fallacy } \\
\text { OnlineStatbook } \\
\text { D.Lane }\end{array}$ & $\begin{array}{l}\text { Experimento aleatorio de lanzamiento de una moneda perfecta. Para } \\
\text { secuencias, que se pueden repetir tantas veces como se quiera, de } 1 \text {, } \\
100 \text { y } 1.000 \text { lanzamientos, se calculan las frecuencias absolutas y rela- } \\
\text { tivas de los sucesos cara y cruz. Para secuencias de } 25.000 \text { lanzamien- } \\
\text { tos se realizan los mismos cálculos y se representan gráficamente tanto } \\
\text { la frecuencia relativa del suceso cara, como la diferencia entre el nú- } \\
\text { mero de caras y de cruces. }\end{array}$ \\
\hline Sim11 & $\begin{array}{l}\frac{\text { Lanzamiento moneda/dado }}{\text { Aula Virtual Estadística }} \\
\text { M. Aguilera }\end{array}$ & $\begin{array}{l}\text { Dos experimentos aleatorios: lanzamiento de una moneda y lanzamiento } \\
\text { de un dado (ambos perfectos o trucados). Los lanzamientos pueden re- } \\
\text { petirse en secuencias de } 1,10,50,100,500 \text { y 1.0oo, con un máximo de } \\
\text { 2.ooo tiradas. Se visualiza la frecuencia absoluta y relativa de los resul- } \\
\text { tados del experimento en forma de tabla, y se representa la evolución de } \\
\text { la frecuencia relativa respecto al número creciente de tiradas. Aparecen } \\
\text { las probabilidades del modelo teórico para su comparación. }\end{array}$ \\
\hline Sim12 & $\begin{array}{c}\text { C1FreqRel } \\
\text { Proyecto Statmedia }\end{array}$ & $\begin{array}{l}\text { Representación de la frecuencia relativa del suceso cara en la secuencia } \\
\text { de } n \text { lanzamientos de una moneda perfecta. El valor de n puede variarse } \\
\text { en un intervalo 1-10oo y observar la diferencia entre la frecuencia re- } \\
\text { lativa y la probabilidad teórica. }\end{array}$ \\
\hline Sim13 & $\begin{array}{l}\frac{\text { Law of Large Numbers: Dice }}{\text { Rolling Example }} \\
\text { Proyecto Demostración Wolfram } \\
\text { P. Savory }\end{array}$ & $\begin{array}{l}\text { Experimento aleatorio de lanzamiento de un dado perfecto. Muestra } \\
\text { las frecuencias relativas de cada uno de los resultados del dado en una } \\
\text { secuencia de n tiradas (máximo 9.950) a través de un diagrama de sec- } \\
\text { tores. La representación es dinámica variando el número de veces que } \\
\text { se lance el dado. Representa también el valor del resultado medio con- } \\
\text { seguido frente al número creciente de tiradas. }\end{array}$ \\
\hline Sim14 & $\begin{array}{c}\frac{1.000 \text { lanzamientos de un dado }}{\text { Geogebra }} \\
\text { M. Sada Allo }\end{array}$ & $\begin{array}{l}\text { Experimento aleatorio de lanzamiento de un dado perfecto. Muestra } \\
\text { las frecuencias relativas de cada uno de los resultados del dado en una } \\
\text { secuencia de n tiradas (máximo 1.ooo tiradas) a través de un diagrama } \\
\text { de barras. La representación es dinámica, variando el número de veces } \\
\text { que se lanza el dado. Aparecen las probabilidades del modelo teórico } \\
\text { para su comparación. }\end{array}$ \\
\hline Sim15 & $\begin{array}{l}\text { Two Dice with Histogram } \\
\text { Proyecto Demostración Wolfram } \\
\text { A. Brown }\end{array}$ & $\begin{array}{l}\text { Experimento aleatorio de lanzamiento de dos dados perfectos. Para se- } \\
\text { cuencias de } 10,50,100,500 \text { y } 1.000 \text { lanzamientos muestra, de forma } \\
\text { dinámica, a medida que crece el número de lanzamientos, el diagrama } \\
\text { de barras de los resultados del experimento, en términos de la suma } \\
\text { de las puntuaciones de los dos dados. }\end{array}$ \\
\hline
\end{tabular}

Tabla 4: Simulaciones interactivas seleccionadas por su utilidad para la consecución del objetivo C 


\begin{tabular}{|c|c|c|}
\hline \multicolumn{2}{|c|}{ SIMULACIÓN INTERACTIVA } & \multirow[b]{2}{*}{$\begin{array}{l}\text { DESCRIPCIÓN } \\
\text { Para dos sucesos. Permite modificar la probabilidad y la relación entre } \\
\text { ambos. Visualiza la probabilidad conjunta como proporción que el espacio } \\
\text { ocupado por el suceso intersección supone sobre el espacio muestral, frente } \\
\text { a la probabilidad condicionada, como proporción que el espacio ocupado } \\
\text { por el suceso intersección supone sobre el suceso condición. }\end{array}$} \\
\hline $\operatorname{Sim} 16$ & $\begin{array}{l}\text { Conditional probability } \\
\text { Proyecto Demostración Wolfram } \\
\text { C. Boucher }\end{array}$ & \\
\hline Sim17 & $\begin{array}{l}\text { Conditional probability } \\
\text { OnlineStatbook } \\
\text { D.Lane }\end{array}$ & $\begin{array}{l}\text { Simulación en la que, sobre un espacio muestral formado por cruces y cír- } \\
\text { culos de diferentes colores, se calculan distintas probabilidades condicio- } \\
\text { nadas a partir del número de elementos favorables al suceso intersección } \\
\text { sobre el número de elementos favorables al suceso condición. }\end{array}$ \\
\hline $\operatorname{Sim} 18$ & $\begin{array}{l}\frac{\text { Dice and conditional probabilities }}{\text { Mathematics for Liberal Arts }} \\
\text { F. Richman et al. }\end{array}$ & $\begin{array}{l}\text { Experimento aleatorio de lanzamiento de dos dados. Sobre una tabla en la } \\
\text { que se visualizan los distintos pares posibles del experimento, representa } \\
\text { en colores diferentes dos sucesos (seleccionados por el usuario entre dis- } \\
\text { tintos predefinidos), así como el suceso intersección entre ambos. Contando } \\
\text { elementos en cada uno de ellos es posible calcular sus probabilidades así } \\
\text { como las probabilidades condicionadas correspondientes. }\end{array}$ \\
\hline
\end{tabular}

Tabla 5: Simulaciones interactivas seleccionadas por su utilidad para la consecución del objetivo D

lando la probabilidad de los sucesos intersección y condición a través de la regla de Laplace (ver Tabla 5).

E. Planteamiento de los experimentos en etapas y el uso de los teoremas de la Probabilidad Total y Bayes para su resolución. Para algunos alumnos el teorema de Bayes resulta complejo ya que para su comprensión se requieren conceptos y propiedades previas que deben tener claros, como son: probabilidades simples, compuestas y condicionales; partición del espacio muestral; suceso y su complemento y el teorema de la Probabilidad Total.

Mediante la construcción del diagrama de árbol, se pueden aclarar algunas de las dudas. En la primera ramificación se consideran los sucesos de la partición y se colocan sus probabilidades. En la segunda ramificación, para cada suceso se considera la probabilidad de que ocurra el suceso que se está buscando. En los puntos finales de las ramificaciones se colocan las probabilidades y al sumar estas probabilidades se obtiene el teorema de la probabilidad Total. En el applet de Argote (Sim19) del proyecto Descartes pueden trabajar el teorema de Bayes desde esta perspectiva. El applet se acompaña de otro en el que pueden experimentar con el Teorema de la Probabilidad Total.

El applet de Borrego en el aula virtual de Estadística
(Sim20) adopta también el enfoque de construcción del diagrama de árbol para trabajar con ambos teoremas, aunque mejora los applets anteriores permitiendo ampliar el número de sucesos en la partición hasta cuatro, y visualizando los conceptos que llevan aparejados estos teoremas. El libro online fruto de Statmedia I, en el caso 3 del tema de Probabilidad, proporciona la posibilidad de experimentar con un juego de dados y urnas clásico para la aplicación de los teoremas (Sim21) (ver Tabla 6).

F. Conexión entre el tema de probabilidad en el que trabajan con sucesos y el concepto de variable aleatoria.

Los alumnos encuentran complicado el paso del tema de fundamentos de probabilidad, en el que representan los resultados de un experimento como sucesos u operaciones con sucesos, al tema de variables aleatorias, en el que se traducen esos resultados a valores cuantitativos. El uso de simulaciones interactivas puede ayudarles también a hacer esa conexión de manera más sencilla.

En los experimentos de lanzamiento de monedas, si se trabaja con más de una, se puede contar, por ejemplo, el número de caras obtenido. Las simulaciones revisadas trabajan con la distribución Binomial resultante de distintas formas. Se puede seguir una aproximación similar a la que se adoptó

\begin{tabular}{|c|c|c|}
\hline \multicolumn{2}{|c|}{ SIMULACIÓN INTERACTIVA } & DESCRIPCIÓN \\
\hline Sim19 & $\begin{array}{l}\frac{\text { Teorema de Bayes }}{\text { Proyecto Descartes }} \\
\text { J.A. Argote }\end{array}$ & $\begin{array}{l}\text { Problema representado por un árbol con dos ramas en dos puntos de rami- } \\
\text { ficación sucesivos en el que se permite modificar las probabilidades de la } \\
\text { primera y la segunda ramificación. Ofrece el cálculo de cuatro posibles pro- } \\
\text { babilidades empleando en todas ellas el teorema de Bayes. }\end{array}$ \\
\hline Sim20 & $\begin{array}{c}\text { Probabilidad total y teorema de Bayes } \\
\text { Aula Virtual Estadística } \\
\text { J. Borrego }\end{array}$ & $\begin{array}{l}\text { Problema representado por un árbol con dos puntos de ramificación suce- } \\
\text { sivos, de forma que en el primero se puede elegir entre dos, tres o cuatro } \\
\text { ramas posibles. Eligiendo espacio muestral continuo, se pueden definir las } \\
\text { probabilidades en los dos puntos de ramificación. Permite y explicita el } \\
\text { cálculo de las probabilidades que utilizan tanto el teorema de la Probabili- } \\
\text { dad Total como el de Bayes. Representa sobre la partición del espacio } \\
\text { muestral de la primera ramificación los sucesos implicados en ambos teo- } \\
\text { remas, lo que facilita la interpretación de las probabilidades resultantes. }\end{array}$ \\
\hline Sim21 & $\begin{array}{l}\frac{\text { Juego de dados y urnas }}{\text { Proyecto Statmedia }} \\
\text { Statmedia I }\end{array}$ & $\begin{array}{l}\text { Experimento de lanzamiento de un dado cuyo resultado condiciona la urna } \\
\text { en la que se realiza una extracción. Se pueden definir tanto las consecuencias } \\
\text { del lanzamiento del dado (con respecto a tres urnas diferentes), como la com- } \\
\text { posición de las urnas (con tres posibles colores en las bolas que contienen). } \\
\text { En el juego hay disponibles dos applets, uno para calcular la probabilidad de } \\
\text { extracción de una bola de cada uno de los colores posibles dada la configura- } \\
\text { ción definida del experimento utilizando el teorema de la Probabilidad Total, } \\
\text { y otro para calcular la probabilidad de estar realizando la extracción en cada } \\
\text { una de las urnas cuando se ha obtenido una bola de un color concreto (tam- } \\
\text { bién a definir entre los tres posibles), a partir del teorema de Bayes. }\end{array}$ \\
\hline
\end{tabular}

Tabla 6: Simulaciones interactivas seleccionadas por su utilidad para la consecución del objetivo $\mathrm{E}$ 
en las figuras 10 a 15 con la interpretación frecuentista de la probabilidad, tal y como hace Sada Allo (Sim22). También se puede trabajar con el aparato de Galton, en el que se simula la distribución Binomial a partir de la caída de bolas que van chocando con un número de filas variable y que presentan una probabilidad preestablecida de tomar uno u otro camino tras el choque. Existen numerosas simulaciones del aparato de Galton, siendo la de Sada Allo (Sim23) un ejemplo de simulación en la que se permiten alterar los dos parámetros de la distribución Binomial resultante, se observa la caída de las bolas de manera dinámica, y se puede representar también el modelo teórico subyacente, lo que resulta útil a efectos de comparación (ver Tabla 7).

También el experimento de lanzamiento de dados, que se ha trabajado desde distintos enfoques, puede utilizarse para completar la conexión con el tema de variables aleatorias. Siguiendo una aproximación similar a la de la simulación de Brown (Sim15), Alvarez y Losada crean, para el proyecto Gauss, un juego (Sim24) en el que el alumno puede apostar en una carrera de caballos donde los movimientos se deciden según la suma conseguida en el lanzamiento de dos dados. El juego tiene otra versión Carrera de caballos (resta) en la que el movimiento se decide en función de la diferencia (en valor absoluto) entre las puntuaciones de los dos dados, por lo que el uso de ambos permite la definición de distintas variables aleatorias en el mismo experimento.

El concepto de esperanza matemática puede trabajarse también con estos applets. El applet de Savory (Sim13) representa además la convergencia de la puntuación media en el lanzamiento de un dado. Por su parte, en el proyecto Statmedia se localiza un applet que simula el juego de apuesta de la primitiva y con el que se puede observar la dificultad de conseguir premios con una determinada apuesta. Este applet calcula el número medio de aciertos en el experimento, y se puede utilizar para que los alumnos comparen los resultados que se van obteniendo a medida que se incrementa el número de juegos con el número medio de aciertos en el modelo teórico subyacente. Este modelo teórico está descrito de una manera muy amena en la web Estadística para todos.

4. Ejemplo práctico de integración de las simulaciones Las simulaciones interactivas pueden utilizarse como apoyo a las clases expositivas, utilizando la pizarra digital o simplemente un cañón de vídeo, de forma que nos ayuden a clarificar conceptos estadísticos a través de su visualización. También pueden emplearse como apoyo a las actividades prácticas, ya que podemos incorporarlas a las actividades que diseñamos, tanto a las que cuentan con la presencia del profesor, como las que se diseñan para realizarse de forma no presencial.

Nuestra experiencia con el uso de las simulaciones en la docencia advierte del riesgo que supone presentarlas a los alumnos (mostrarles dónde se encuentran, qué proporcionan y cómo se usan) y dejar a su libre albedrío el uso de las mismas como parte de los materiales disponibles. El resultado más probable cuando se sigue esta estrategia es que sea sólo un número muy reducido de estudiantes el que finalmente las emplea.

Creemos que es labor del profesor la integración de las mismas en actividades que aprovechen todo su potencial, de forma que verdaderamente mejoren el proceso de aprendizaje de nuestros alumnos. En esa incorporación de las simulaciones:

- debe intentarse que el número de simulaciones que se propone no sea muy elevado (riesgo que se corre dado el gran número de recursos disponibles), por lo que hay que valorar lo que se obtiene con cada una de ellas.

- debe prepararse el momento de su uso, provocando en los alumnos, a través de preguntas previas, la elaboración de una primera idea que será contrastada a través de la simulación.

- debe comprobarse si, con el uso de las simulaciones, mejora el proceso de aprendizaje de los temas tratados.

A modo de ejemplo, una actividad práctica de incorporación de las simulaciones ${ }^{2}$, puede tener una estructura en la que se distingan varias partes:

Parte 1: completar ejercicios similares a los que planteamos habitualmente en las relaciones o en los manuales de problemas.

Parte 2: revisar las cuestiones planteadas utilizando las simulaciones interactivas.

Parte 3: rellenar una hoja de conclusiones mostrando si han cambiado la respuesta inicial y por qué, y contestar a cuestiones complementarias que persiguen comprobar si se han afianzado los conceptos.

\begin{tabular}{|c|c|c|}
\hline \multicolumn{2}{|c|}{ SIMULACIÓN INTERACTIVA } & DESCRIPCIÓN \\
\hline Sim22 & $\begin{array}{c}\frac{\text { Lanzando cuatro monedas }}{\text { Geogebra }} \\
\text { M. Sada Allo }\end{array}$ & $\begin{array}{l}\text { Experimento aleatorio de lanzamiento de cuatro monedas perfectas. Con- } \\
\text { siderando como resultado del experimento el número de caras obtenidas, } \\
\text { muestra las frecuencias relativas de cada uno de los posibles resultados } \\
\text { cuando el experimento se repite n veces (máximo } 200 \text { repeticiones) a través } \\
\text { de un diagrama de barras. La representación es dinámica, variando el nú- } \\
\text { mero de veces que se lanzan las monedas. Aparecen las probabilidades del } \\
\text { modelo teórico para su comparación. }\end{array}$ \\
\hline Sim23 & $\begin{array}{c}\frac{\text { El aparato de Galton }}{\text { Geogebra }} \\
\text { M. Sada Allo }\end{array}$ & $\begin{array}{l}\text { Aparato de Galton en el que se simula el lanzamiento de } 200 \text { bolas, pu- } \\
\text { diendo configurar tanto el número de filas del aparato (entre } 2 \text { y 12) y la } \\
\text { probabilidad de desplazarse a uno de los dos lados después del choque. Se } \\
\text { pueden realizar experimentos diferentes y se muestran las probabilidades } \\
\text { del modelo teórico para su comparación. }\end{array}$ \\
\hline Sim24 & $\begin{array}{c}\frac{\text { Carrera de caballos (suma) }}{\text { Proyecto Gauss }} \\
\text { J.M. Alvarez y R. Losada }\end{array}$ & $\begin{array}{l}\text { Juego basado en el experimento aleatorio de lanzamiento de dos dados per- } \\
\text { fectos. Hay doce caballos por los que se puede apostar numerados del } 1 \text { al } 12 . \\
\text { El juego consiste en apostar por uno de ellos y observar cómo se va realizando } \\
\text { el desplazamiento, ya que se desplaza cada vez el caballo cuyo dorsal coincida } \\
\text { con la suma de las puntuaciones de los dos dados. }\end{array}$ \\
\hline
\end{tabular}

Tabla 7: Simulaciones interactivas seleccionadas por su utilidad para la consecución del objetivo $\mathrm{F}$

${ }^{2}$ Un ejemplo práctico y completo de incorporación de las simulaciones interactivas en el tema de fundamentos de probabilidad puede encontrarse en Arnaldos y Faura (2011). 


\begin{tabular}{|l|l|}
\hline OBJETIVO & SIMULACIONES INTERACTIVAS \\
\hline $\begin{array}{l}\text { Comprensión de los sucesos, las operaciones con } \\
\text { los mismos y las consecuencias de los axiomas de }\end{array}$ & $\begin{array}{l}\text { Venn diagram applet (Sim1) } \\
\text { Interactive Venn Diagrams (Sim2) } \\
\text { Diagrama de Venn (Sim6) }\end{array}$ \\
\hline $\begin{array}{l}\text { Cálculo de probabilidades mediante la interpre- } \\
\text { tación clásica de la probabilidad e interpretación } \\
\text { del concepto de probabilidad condicionada }\end{array}$ & $\begin{array}{l}\text { C1BasicDie (Sim7) } \\
\text { Condicional Probability Applet (Sim4) } \\
\text { Conditional probabilities (Sim8) }\end{array}$ \\
\hline $\begin{array}{l}\text { Comprensión del significado de la interpretación } \\
\text { frecuentista de la probabilidad }\end{array}$ & $\begin{array}{l}\text { Lanzamiento moneda/dado (Sim11) } \\
\text { Planteamiento de los experimentos en etapas y el } \\
\text { uso de los teoremas de la Probabilidad Total y } \\
\text { Bayes para su resolución }\end{array}$ \\
\hline
\end{tabular}

Tabla 8: Ejemplo de selección de simulaciones interactivas

En función de la programación del tema dentro del conjunto de la materia a impartir, la selección de ejercicios y simulaciones será diferente. La Tabla 8 muestra una de las posibles combinaciones que se puede utilizar, aunque las posibilidades son numerosas y vienen dictadas por los objetivos que se pretendan conseguir, los ejercicios que se planteen y la mayor o menor medida en que las simulaciones interactivas sean configurables para reproducir la situación descrita en los ejercicios.

\section{Conclusiones}

Existe gran cantidad de recursos en la red (libros online, revistas educativas, proyectos de visualización,...) que pueden ser utilizados como una herramienta más en la docencia de las asignaturas de Estadística y que pueden mejorar y facilitar el proceso de aprendizaje y formación. El empleo de las TICs, y en concreto de las simulaciones interactivas, permite al estudiante adquirir o mejorar competencias, haciendo que el proceso de enseñanza-aprendizaje sea más ameno y atractivo.

En nuestras clases las hemos integrado tanto como complemento a la lección magistral usando las pizarras digitales, como acompañando a los ejercicios tradicionales. Estas herramientas se pueden utilizar tanto en actividades no presenciales, como proceso de autoaprendizaje y autoevaluación, como en actividades presenciales, en las que el papel del profesor es más importante. La labor del profesor cuando se quiere integrar este recurso en la docencia debe ser discriminar entre todos los recursos existentes, atendiendo a los objetivos que se persigan, motivar correctamente a los alumnos en su uso y valorar la eficacia del empleo de las mismas.

Realizada la experiencia de integración de las TICs en el ámbito de la probabilidad durante varios cursos académicos, hemos podido comprobar el alto nivel de satisfacción tanto del profesorado como de los alumnos. Un aspecto importante ha sido una mayor motivación en el alumnado, aunque no siempre ha llevado consigo una mejora en su rendimiento. Somos conscientes que la incorporación de las TICs no significa necesariamente una docencia más eficiente y mayor calidad en el aprendizaje de los estudiantes. Para conseguirlo, es necesario planificar y diseñar muy bien cuándo y cómo deben integrarse las TICs, seleccionando aquellos recursos que se adecuen a los objetivos buscados.

Este es sólo un ejemplo de incorporación de las simulaciones interactivas en Estadística. Hemos realizado otras experiencias de integración de éstas en otras partes de la Estadística, como Estadística Descriptiva, Modelos de variables aleatorias o Inferencia Estadística, obteniendo también resultados satisfactorios.

\section{Bibliografía}

Aguilera, Marisa. Lanzamiento moneda/dado. En Aula virtual de Estadística (Universidad de Córdoba). Jurado Bello, Manuel, Diz Pérez, José y Espejo Mohedano, Roberto (coordinadores). http://www.uco.es/simulaciones estadisticas/set/MarisaAguilera/MonedaDado/?idioma=es. Fecha de consulta, 20/07/2011.

Alvarez García, Jose Luis, y Losada Liste, Rafael. Carrera de caballos (resta). Simulación. Proyecto Gauss. Ministerio de Educación.

http://recursostic.educacion.es/gauss/web/materiale$\underline{\mathrm{s} \text { didacticos/eso/actividades/estadistica y probabili- }}$ dad/estimacion/carrera caballos resta simula/activi dad.html. Fecha de consulta, 20/07/2011.

Alvarez García, Jose Luis, y Losada Liste, Rafael. Carrera de caballos (suma). Simulación. Proyecto Gauss. Ministerio de Educación.

http://recursostic.educacion.es/gauss/web/materiales didacticos/eso/actividades/estadistica y probabilidad/estimacion/carrera caballos suma simula/activi dad.html. Fecha de consulta, 20/07/2011.

Argote Martín, Juan Alberto. Teorema de Bayes. Proyecto Descartes. Ministerio de Educación. http://recursostic.educacion.es/descartes/web/materiales didacticos/probabilidad condicionada/probabilidad bayes jam.htm. Fecha de consulta, 20/07/2011.

Argote Martín, Juan Alberto. Teorema de la Probabilidad Total. Proyecto Descartes. Ministerio de Educación. http://recursostic.educacion.es/descartes/web/materi ales didacticos/probabilidad condicionada/probabilidad total jam.htm. Fecha de consulta, 20/07/2011.

Arnaldos García, F. y Faura Martínez, U. (2011). Cómo mejorar la comprensión de los fundamentos de la probabilidad a través de las TICs. III Jornadas de Docencia en Economía. Cartagena.

Barrow, Ron F. Conditional probabilities. http://www.waldomaths.com/Prob2N.jsp. Fecha de consulta, 16/12/2012.

Beck, George y Kent, Liz. Venn Diagrams. Proyecto Demostración Wolfram. http://demonstrations.wolfram.com/VennDiagrams/. Fecha de consulta, 20/07/2011.

Borrego, Jesús. Diagrama de Venn. En Aula virtual de Estadística (Universidad de Córdoba). Jurado Bello, Manuel, Diz Pérez, José y Espejo Mohedano, Roberto (coordinadores). http://www.uco.es/simulaciones estadisticas/set/JesusBorrego/DiagramadeVenn/?idioma=es. Fecha de consulta, 20/07/2011. 
Borrego, Jesús. Teorema de la probabilidad total y Teorema de Bayes. En Aula virtual de Estadística (Universidad de Córdoba). Jurado Bello, Manuel, Diz Pérez, José y Espejo Mohedano, Roberto (coordinadores). http://www.uco.es/simulaciones estadisticas/set/Jes usBorrego/ProbabilidadTotal/?idioma=es. Fecha de consulta, 20/07/2011.

Boucher, Chris. Conditional probability. Proyecto Demostración Wolfram.

http://demonstrations.wolfram.com/ConditionalProbability/. Fecha de consulta, 20/07/2011.

Brodie, Marc. Interactive Venn Diagrams. Proyecto Demostración Wolfram. http://demonstrations.wolfram.com/InteractiveVennDiagrams/. Fecha de consulta, 20/07/2011.

Brown, Abby. Two Dice with Histogram. Proyecto Demostración Wolfram.

http://demonstrations.wolfram.com/TwoDiceWithHistogram/. Fecha de consulta, 20/07/2011.

Calvo i Llorca, Miquel (coordinador). Proyecto Statmedia. Universidad de Barcelona. Applets del tema de probabilidad: C1BasicDie, C1BasicUrns, C1FreqRel y C1LotPrim. http://www.ub.edu/stat/GrupsInnovacio/Statmedia/d emo/applets/appletso1es.htm. Fecha de consulta, 20/07/2011.

Calvo i Llorca, Miquel (coordinador). Proyecto Statmedia. Universidad de Barcelona. Demostración online del proyecto Statmedia I.

http://www.ub.edu/stat/GrupsInnovacio/Statmedia/d emo/Statmedia.htm. Fecha de consulta, 20/07/2011.

Cano Villalba, Marisa y Gras Martí, Albert (2006). Tic@'t: una metodología no presencial para el aprendizaje de herramientas TIC y la formación interdisciplinar para toda la comunidad educativa. Revista ieRed: Revista Electrónica de la Red de Investigación Educativa [en línea], 1 (4), pp.1-17.

http://revista.iered.org/v1n4/pdf/mcyag.pdf. Fecha de consulta, 20/07/2011.

Dinov, Ivo D., Christou, Nicolas y Gould, Robert (2009). Law of Large Numbers: the Theory, Applications and Technology-based Education. Journal of Statistics Education, 17 (1).

http://www.amstat.org/publications/jse/v17n1/dinov.

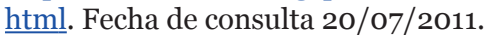

Gámez Mellado, Antonio y Marín Trechera, Luis Miguel (2010). Distribuciones estadísticas: un ejemplo de uso de GeoGebra en enseñanza universitaria. Epsilon 74, pp. 33-42.

Gisbert Cervera, Mercé, Cela-Ranilla, José M. y Isus Barado, Sofía (2010). Las simulaciones en entornos TIC como herramientas para la formación en competencias transversales de los estudiantes universitarios. TESI, 11 (3), pp.352-370.

Giuliano, Mónica, Pérez, Silvia N. y Sacerdoti, Aldo (2011). Inclusión de tecnologías de la información y comunica- ción en la formación estadística. @tic. revista d'innovació educativa, 6, pp.1-9.

http://ojs.uv.es/index.php/attic/article/view/290.

Fecha de consulta, 14/05/2011.

Lane, David. Conditional probability. Online Statistics Education: An Interactive Multimedia Course of Study. http://onlinestatbook.com/simulations/conditional p Lconditional p.html. Fecha de consulta, 20/07/2011.

Lane, David. Gamblers Fallacy. Online Statistics Education: An Interactive Multimedia Course of Study. http://onlinestatbook.com/simulations/gambler falla cy/gambler.html. Fecha de consulta, 20/07/2011.

Richman, Fred, Walker, Carol L., Wisner, Robert J. y Brewer, James W. Mathematics for Liberal Arts. Dice and conditional probabilities.

http://math.fau.edu/richman/Liberal/dice.htm. Fecha de consulta, 20/07/2011.

Rodríguez Izquierdo, Rosa María (2010). El impacto de las TIC en las transformaciones de la enseñanza universitaria: repensar los modelos de enseñanza y aprendizaje. TESI, 11 (3), pp.32-68.

Romero, Victoria L., Berger, Dale E., Healy, Michael R. y Aberson, Christopher L. (2000). Using cognitive learning theory to design effective on-line statistics tutorials. Behaviour Research Methods, Instruments \& Computers. 32 (2), pp. 246-249.

Sada Allo, Manuel. 1.00o lanzamientos de un dado. http://docentes.educacion.navarra.es/msadaall/geoge bra/figuras/dado.htm. Fecha de consulta, 20/07/2011.

Sada Allo, Manuel. El aparato de Galton. http://docentes.educacion.navarra.es/msadaall/geoge bra/figuras/azar galton.htm. Fecha de consulta, 20/07/2011.

Sada Allo, Manuel. Lanzando cuatro monedas. http://docentes.educacion.navarra.es/msadaall/geogebra/figuras/azar monedas4.htm. Fecha de consulta, 20/07/2011.

Savory, Paul. Law of Large Numbers: Dice Rolling Example. Proyecto Demostración Wolfram. http://demonstrations.wolfram.com/LawOfLargeNumbersDiceRolli ngExample/. Fecha de consulta, 20/07/2011.

Shepperd, Mike. Venn Diagram Applet. http://www.nku.edu/ longa/classes/mat385 resourc es/venn/VennGame.html. Fecha de consulta, 16/12/2012.

Stark, Philip B. Venn diagrams (2 subsets). http://www.stat.berkeley.edu/ stark/Java/Html/Ven n.htm. Fecha de consulta, 20/07/2011.

Stark, Philip B. Venn diagrams (3 subsets). http://www.stat.berkeley.edu/ stark/Java/Html/Ven n3.htm. Fecha de consulta, 20/07/2011.

West, R. Webster. Conditional Probability Applet: http://www.stat.tamu.edu/ west/applets/Venn1.htm. Fecha de consulta, 20/07/2011. 